\title{
Becoming a long term survivor of AIDS involved 5 dimensions of adapting
}

\author{
Barroso J. Reconstructing my life: Becoming a long-term survivor of AIDS. Qual Health Res 1997 Feb;7:57-74.
}

Objective

To describe the experiences of long term survivors of AIDS.

\section{Design}

An ethnographic study.

\section{Setting}

Florida, USA.

\section{Participants}

20 English speaking participants ( $70 \%$ men; $90 \%$ white) aged $\geqslant$ 18 years, diagnosed with AIDS for $\geqslant 3$ years ( 2 participants were diagnosed for only $2 \mathrm{y}$ ), and self identified as long term survivors of AIDS were recruited through fliers in primary health care agencies and by word of mouth.

\section{Methods}

Open ended interviews were conducted over $\geqslant 1$ session in the participant's or researcher's home. Total interview length ranged from 1-6 hours; any one interview was limited to 2 hours. Interviewers began with the statement "tell me about being a long term survivor of AIDS", giving planned prompts as needed. Redundancy of descriptions occurred after 12 participants. Sessions were audiotaped and transcribed verbatim. Transcripts were analysed using content analysis and constant comparison techniques.

\section{Main results}

Participant responses were categorised into 5 overlapping dimensions of adapting.

The dimension of normalising involved the cognitive strategies of maintaining a daily routine, realising that AIDS was episodic, and not allowing AIDS to become one's life focus.

Focusing on living involved the attitudinal strategies of maintaining a positive outlook, planning for the future, and having a focus for one's energy.

Taking care of oneself focused on behaviours to enhance physical and mental health including stopping negative habits (eg, alcohol or drug use), undertaking health promoting activities, taking responsibility for one's health, and decreasing stress.

Being in relation to others required restructuring of the social network by dealing with one's family, renegotiating friendships, helping others with HIV, and developing a relationship with a higher power.

Triumphing, or transcending the illness, required existential strategies that included believing that having AIDS was a blessing, re-establishing life priorities, discovering one's true self, and believing that one could live with AIDS.

\section{Conclusion}

Being a long term survivor of AIDS involved 5 dimensions of adapting which facilitated the reconstruction of participants' lives within the context of AIDS.

Sources of funding: Florida Nurses Foundation and University of Tampa.

For article reprint: Dr J Barroso, 921 West Braddock, Tampa, FL 33603, USA. Fax +1 813972 8495 .

\section{Commentary}

The work presented by Barroso provides an important in depth view of the experiences of long term AIDS survivors. The study results are consistent with other long term survival studies and with previous findings on coping with HIV and AIDS. Barroso's work shows the ability of survivors to view a life threatening diagnosis as a challenge rather than a threat. People who "beat the odds" perceived they did so by focusing on the positive rather than the negative. As such, the study results point to areas of possible intervention for nurses.

Caution, however, must be exercised: the strategies described might help patients find productive and successful ways of adapting to this illness, but those strategies may not be responsible for survival. In other words, although patients perceived that these strategies enabled them to be long term survivors, this was not necessarily the case. The link between psychosocial factors and survival involves complex immune responses that are still poorly understood. ${ }^{1}$ Studies such as this do not allow us to conclude that using these strategies will lead to becoming long term survivors of AIDS. More work needs to be done to identify the specific ways that nurses can support the development of such attitudes and cognitions.
This study offers nurses directions for providing support to patients living with AIDS, and also for the development and testing of well defined nursing interventions aimed at maintaining optimum health while living with HIV and AIDS.

France Bouthillette, RN, DNS
Head, Nursing Research,
St.Paul's Hospital,
Vancouver, British Columbia, Canada

1 Goodkin K, Mulder CL, Blaney $\mathrm{N}$, et al. Psychoneuroimmunology and human immunodeficiency virus type 1 infection revisited. Arch Gen Psychiatry 1994;51:246-8. 